American Journal of Psychiatric Research and Reviews
(ISSN:2637-479X)

\title{
Long COVID-19 syndrome precaution and management
}

\section{Abdel-Fattah HMM}

Neuropsychiatry Doctor, El-Abbassia Mental Hospital, Cairo, Egypt.

\begin{abstract}
COVID-19 virus defined as illness caused by a novel coronavirus which first discovered in Wuhan City, China. And On January 30, 2020 , the WHO awarded the global health emergency. This is a review of COVID-19's highly affects on almost all the organs and how we precaution and management as the COVID-19 causes many systemic abnormalities like inflammation, endotheliitis, vasoconstriction, hypercoagulability, edema and Lymphocytopenia, with elevated D-dimer, and disseminated intravascular coagulation. Deep vein thrombosis and venous, thrombosis with pulmonary embolization, systemic and pulmonary arterial thrombosis, embolism are reported, ischemic stroke changes, and myocardial infarction are reported also. it can lead to acute coronary syndrome, with heart failure and myocarditis, arrhythmias. Kidney affection was usually secondary to systemic allover disturbances. Stroke may occurred. Delirium and seizures symptoms are common. impaired the tastes are reported with Psychological disturbances are commonly, Lactate dehydrogenase may be elevated. Many skin manifestations including patchy erythematous rash are noticeable, also effects in sexual function and in vitro fertilization success.
\end{abstract}

One of the biggest barriers standing in the way of ending the pandemic the misinformation about the COVID-19 vaccines. At this critical time the Demand for vaccines very effective as it rigorously tested and found to be safe, also Vaccines have no effect on recipients' genetic material and not effect on fertilization also Antibodies from are estimated to last two to four months, so those who have had a previous infection still get this vaccinated. Keywords: Long covid-19, acute kidney injury, acute respiratory distress syndrome, encephalitis, psychosis, sexual dysfunction, In Vitro fertilization,vaccination.
*Correspondence to Author:

Dr. Hesham Maged Mohammed Abdelfatah

Neuropsychiatry Doctor, Egyptian Ministry of Health, El-Abbassia Mental Hospital, Cairo, EGYPT

How to cite this article:

Abdel-Fattah HMM. Long COVID-19 syndrome precaution and management. American Journal of Psychiatric Research and Reviews, 2021; 4:28.

\section{eScîPub}

eSciPub LLC, Houston, TX USA.

Website: https://escipub.com/ 


\section{Abbreviation:}

Acute Kidney Injury (AKI)

post-traumatic stress disorder (PTSD)

Erectile dysfunction ( ED )

in vitro fertilization (IVF)

\section{Introduction}

The COVID-19 It has rapidly spread all around the continents courses, many affected people's and many death. On May 11, 2021, 11:52 GMT The total COVID-19 cases was about 160 million people and number of deaths was about 3 million and half people and the number of recovery was about 137 million and half with active case in this time was about 19million cases divided between $99.4 \%$ mild cases and $0.6 \%$ critical cases, sources: Worldometer most system becoming affects is the respiratory system, However, In critically patients, multiple systems are affected. The COVID-19 binds angiotensin converting enzyme 2 receptors that's receptors found in the vascular endothelial cells, of the body organs, ${ }^{[1]}$. Disturbances of coagulation process and vascular endothelium are contribute to the injury of multiple organs. Cardiac and renal is commonly affected among the patients who die. Injury and damage to the organs may long lasting after the acute infection has disappeared. Different organs may be affected at different times. Long Coved is defined by The National Institute for Health and Care Excellence, as the presence of symptoms that develop during or following an infection with COVID-19 which continue for about 12-weeks or more and are not explained by an alternative diagnosis. This includes both ongoing symptomatic COVID-19 (from 4 to 12 weeks) and "Post-COVID

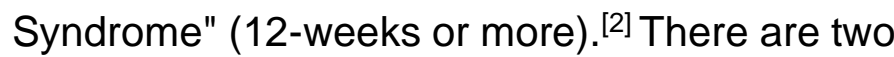
stages known as Long COVID:Ongoing symptomatic COVID-19, that's symptoms last 412 weeks.

\section{Discussion}

COVID-19 lead to production of cytokines by the white blood cells [2]. This abnormal response lead to systemic inflammatory processes, and acute respiratory, with multi-organ damages, shock, and finally death.

Inflammatory process may increased even when the viral load of infection is decreased.COVID19 infects origin endothelial cells and leading to diffuse and massive lymphocytic endotheliitis, which cause vasoconstriction [3].

\subsection{Effect on the liver and GIT}

The liver increases secretion of procoagulant substances. C-reactive protein was elevated. Cytokine storm and massive systemic inflammation are associate with lymphocytopenia, that causes elevates in the Ddimer, which was a prognostic. G.I.T effects: symptoms likely loss of appetite, nausea, vomiting, diarrhea. Figure (1)

\subsection{Precaution and Managemet:}

We recommend thromboprophylaxis ${ }^{[4,5]}$. or anticoagulants oral form, as the Heparins bind tightly to the spike COVID-19 proteins stopping the entry of the virus into cells and also acted as downregulate the immune activation also GIT symptomatic treatments.

2.1 Effect on Chest: in the acute stage the patients have classic diffuse alveolar damage without organization and fibrosis ${ }^{[6,7]}$. It is caused by disruption of endothelial, alveolar cells. This leads to fluid and cellular exudation, Acute fibrinous and pneumonia are also observed [8].On chest CT, findings of subpleural and peripheral areas of ground-glass opacity (G.G.O) and consolidation are present in patients with COVID-19. ${ }^{[9,10]}$, Figure ( 2 )

\subsection{Precaution and Management:}

Several ways are available for managing respiratory insufficiency ${ }^{[9,10]}$. As Oxygen via high-flow nasal cannula, and noninvasive ventilators. British Thoracic Society guidelines define the target range for oxygen saturation as $94-98 \%$ and a level of $92 \%$ or below as requiring supplementary oxygen [11]

\subsection{Effect on heart can occur in the absence} of pulmonary and other complications ${ }^{[12,13]}$. Ischemic injury can occur in patients with coronary artery disease, the primary cause of AJPRR:https://escipub.com/american-journal-of-psychiatric-research-and-reviews/ 2 
the former two is plaque rupture and thrombosis. and treatment the patients COVID-19 and may Figure ( 3 )

\subsection{Precaution and Management:}

Emergency clinicians should be aware of those admit to ICU unit and may usage the mechanical circulatory system in cardiogenic or mixed shock.

cardiovascular complications when evaluating

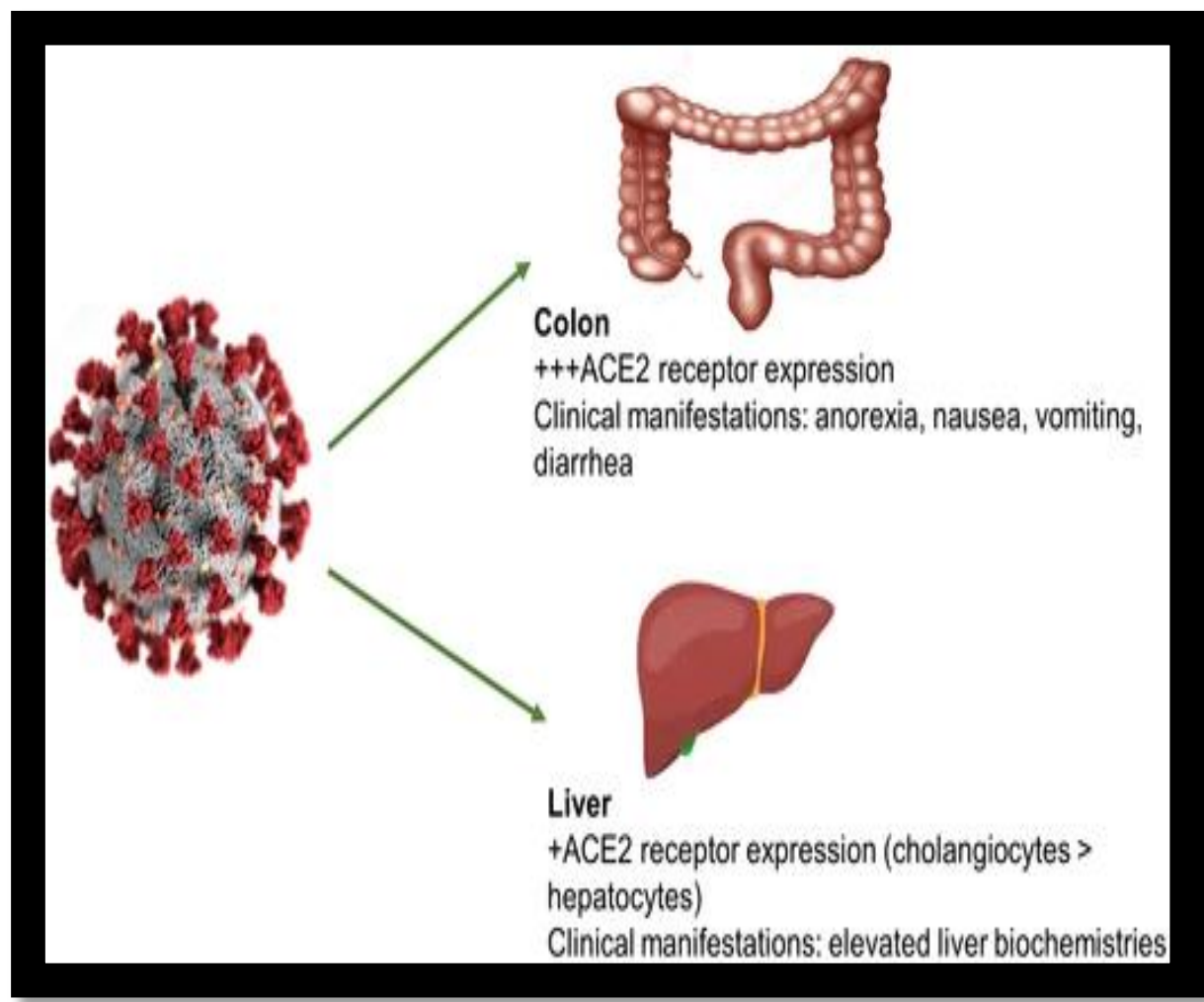

Figure 1 ( Liver injury in the setting of COVID19-related illness)

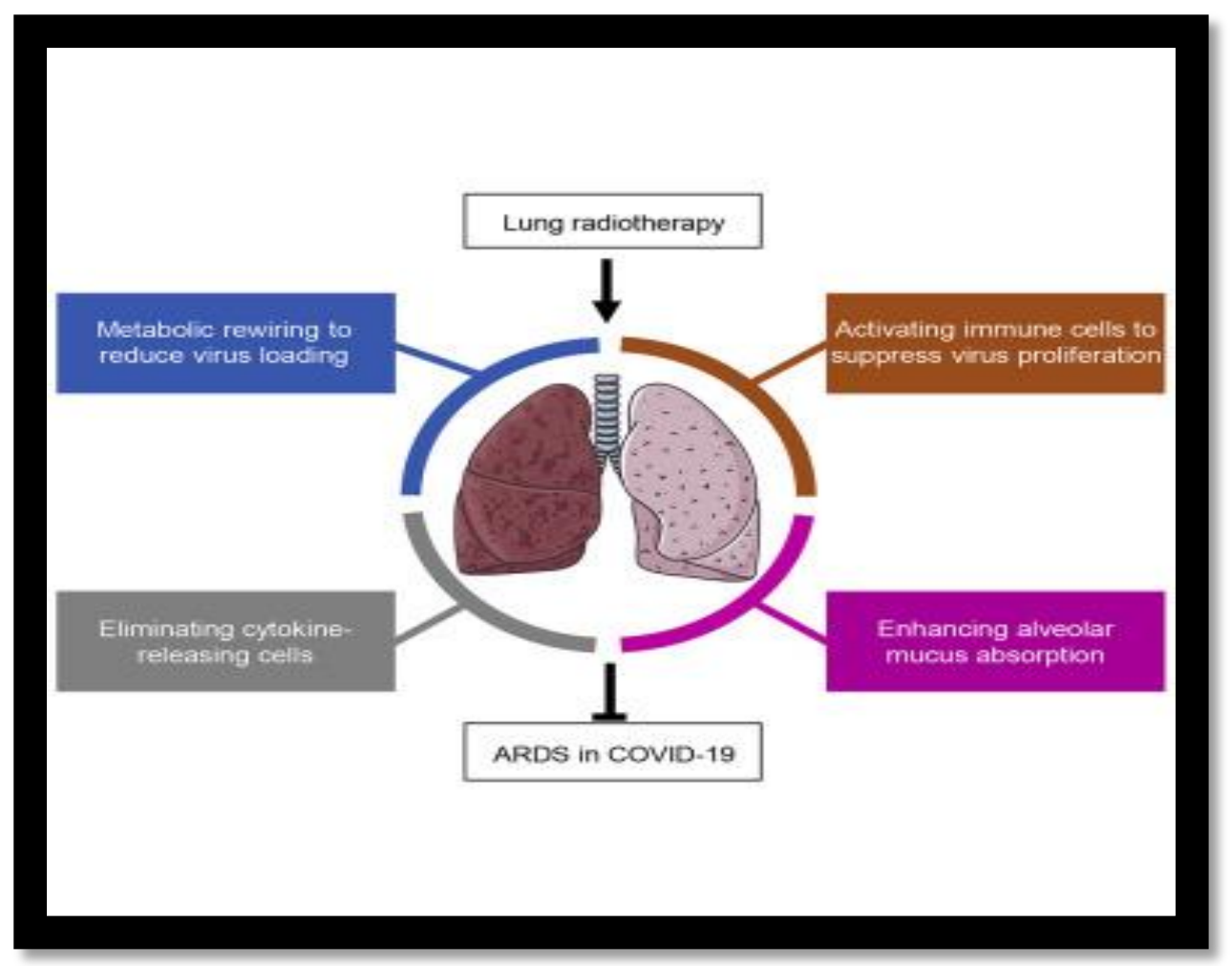

Figure 2 (Coronavirus Induced Acute Respiratory Distress Syndrome) 


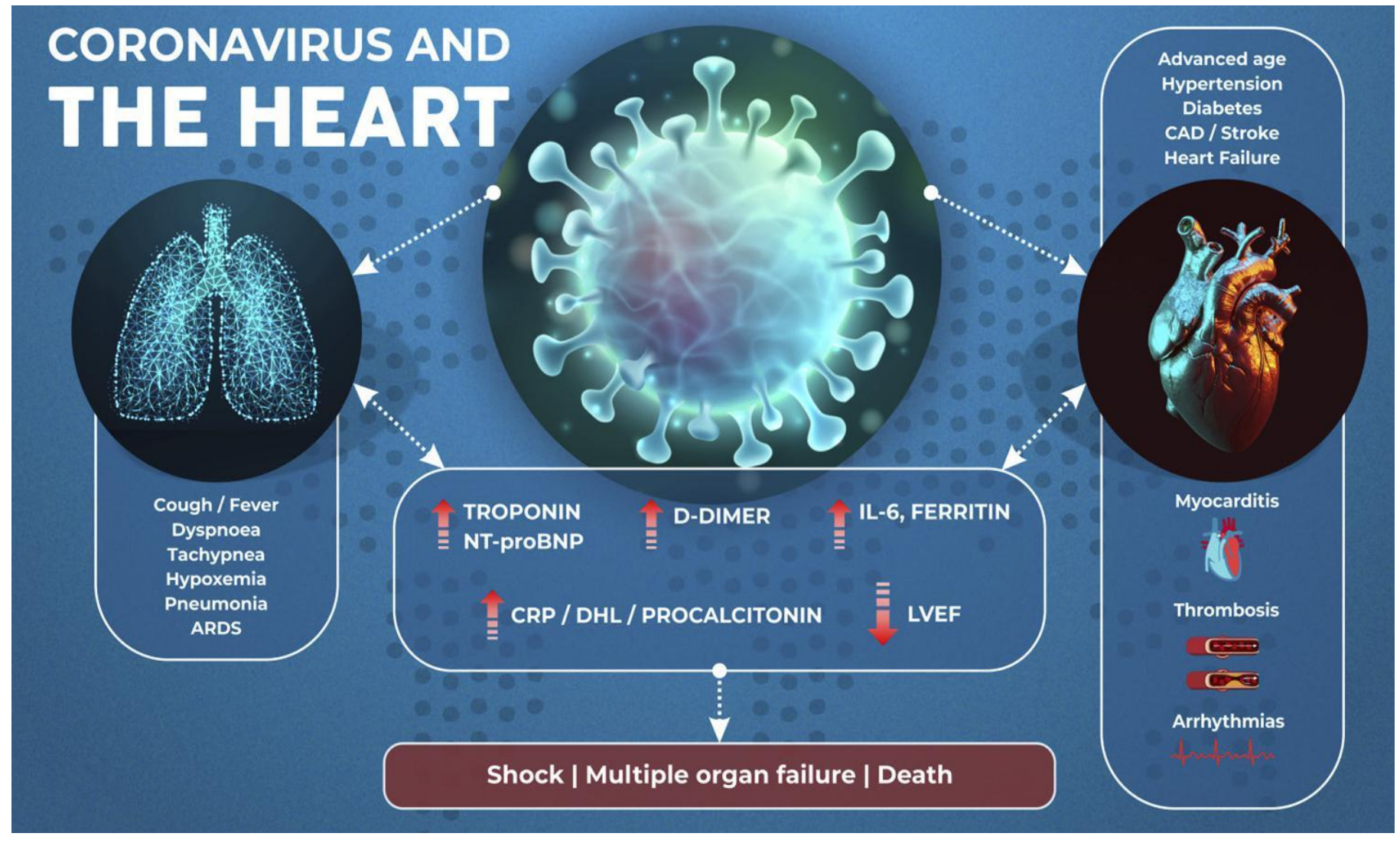

Figure 3 ( Coronavirus and the Heart)

\subsection{Effect on kidney:}

COVID-19 complicates the treatment of patients on dialysis ${ }^{[14]}$. The virus is found in glomerular cells, tubular epithelium, Acute kidney injury (AKI) is commonly secondary to systemic disturbances including diabetes, hypertension, and coagulopathy. Cytokine storms can cause drastic hypoperfusion. Acute kidney injury is also caused due to hyperventilation or medications including antivirals.

\subsection{Precaution and Management:}

individualized fluid and haemodynamic management based on dynamic assessment of cardiovascular status for critically ill patients with COVID-19. and balanced crystalloids as initial management for the expansion of intravascular volume in patients at risk of AKI or with AKI unless a specific indication exists for the use of other fluids.

\subsection{Effect on brain:}

meningitis, encephalitis indicating viral invasion of the CNS. Neurological manifestationcan occur with respiratory or other symptoms impairment [15-17]. Symptoms like dizziness, headache, impaired consciousness, contusion status, delirium, and cognitive impairment.Also the Cytokine storm can cause brain inflammation with edema causing seizure-like. Brain Stroke may found due to thrombus in the cerebral artery ${ }^{[18]}$. Cerebral hemorrhage, Ataxia and seizure also may found. Cranial nerves impairment may also found. [19]. Figure ( 4 )

\subsection{Precaution and Management:}

Proper investment and careful monitoring of CNS symptoms with Perform aerobic exercise to improve brain health and healthy diet including olive oil, fruits and vegetables, nuts and beans, and whole grains has been proven to improve thinking, memory. and brain health with Avoiding alcohol and drugs also Sleep well so the brain and body can clear out toxins and work toward healing.

\subsection{Effect on the eyes:}

About $30 \%$ of hospitalized patients develop

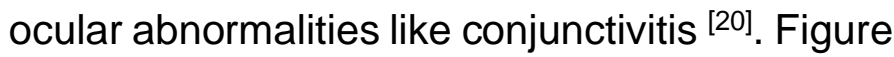
( 5 ) 


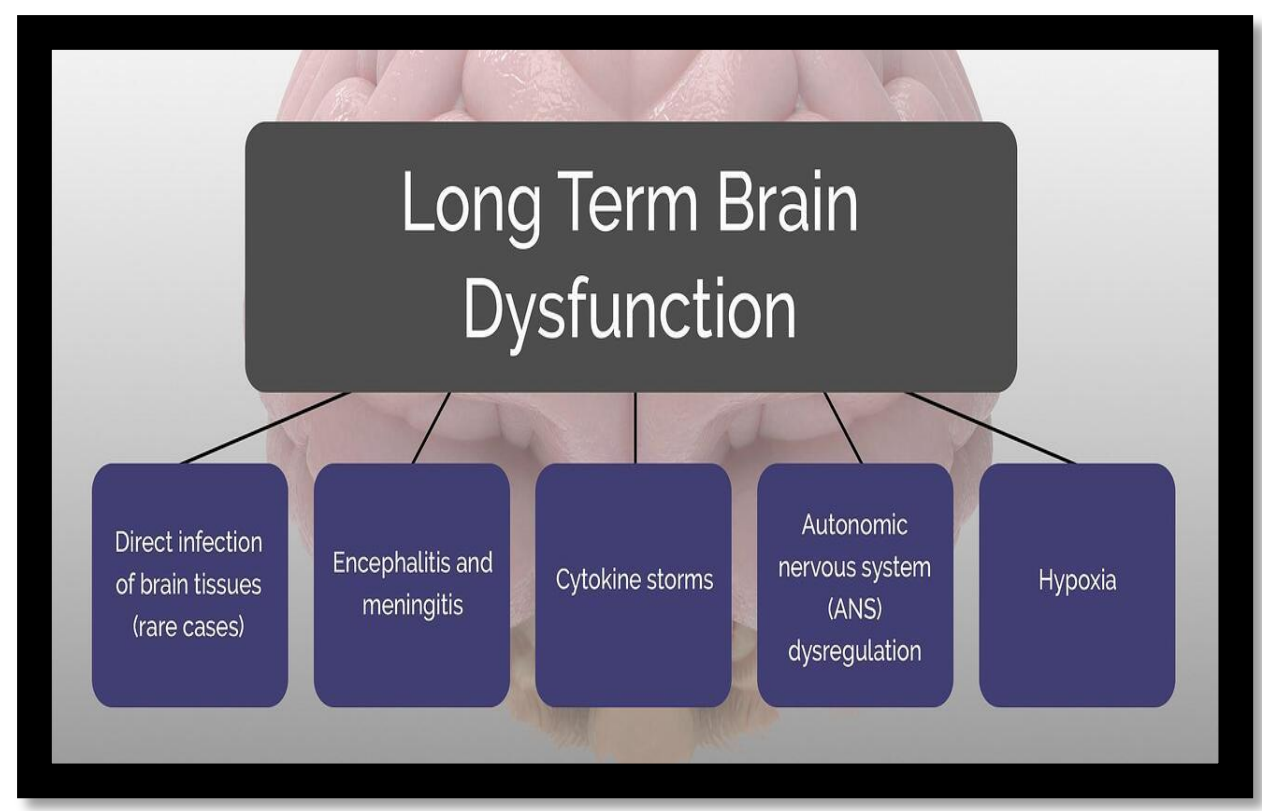

Figure 4 ( Long term brain dysfunction in patients with COVID-19)

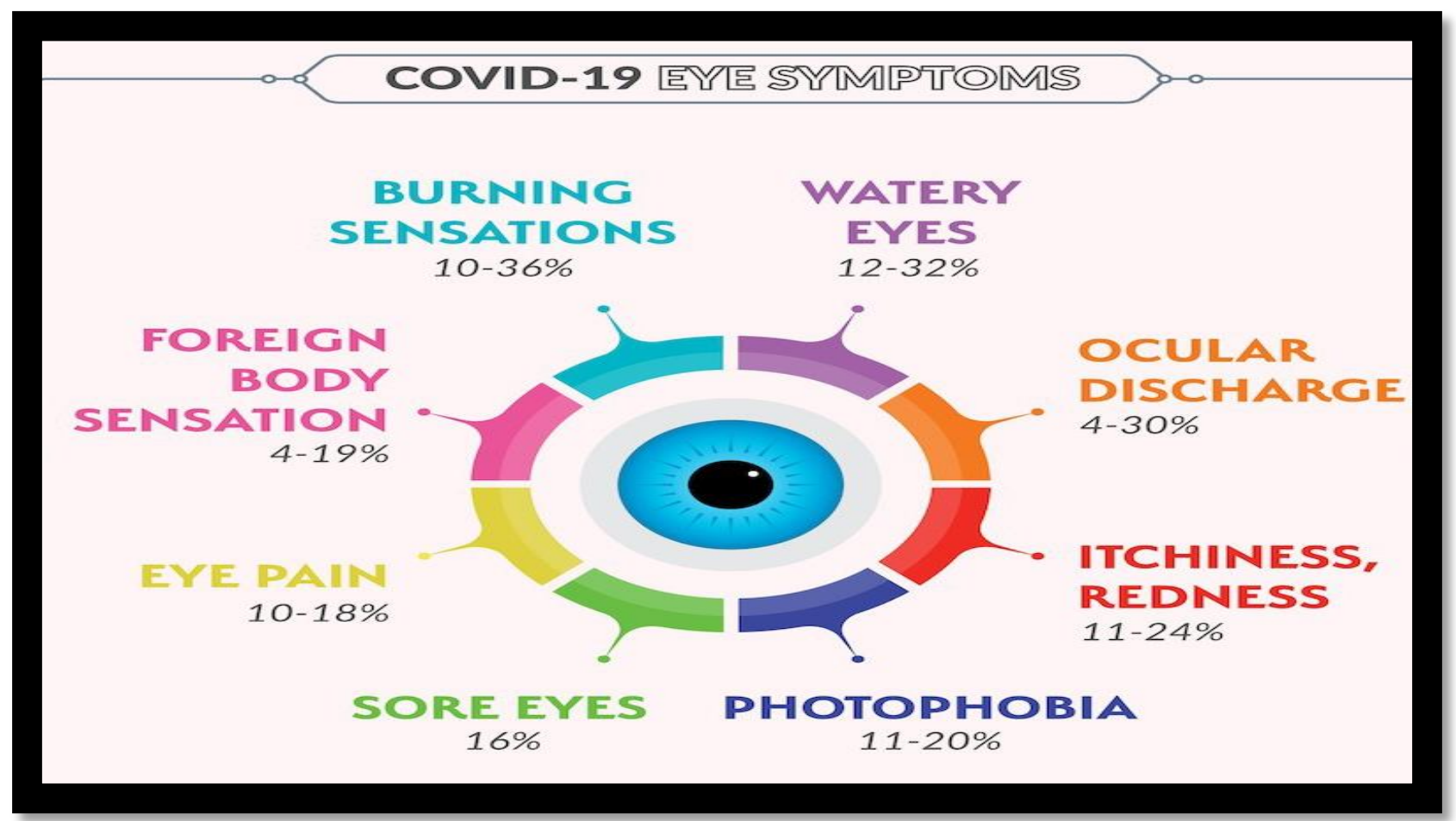

Figure 5 ( COVID-19 Eye Symptoms)

\subsection{Precaution and Management:}

As of March 18, 2020, the American Academy of Ophthalmology has urged all ophthalmologists to produce only urgent or emergent care to scale back the danger of SARS-CoV-2 transmission and to conserve disposable medical supplies. within the absence of great eye pain, decreased vision, or light sensitivity, many patients will be managed remotely with a shot of frequent preservative-free artificial tears, cold compresses, and lubricating ophthalmic ointment. a brief course of topical antibiotics are often added to forestall or treat bacterial superinfection supported the patient's symptoms and risk factors.

\subsection{Effect on skin:}


Skin disorders are likely to other viruses and chronic inflammations like acne or eczema or psoriasis. the majority have patchy erythematous rash [21]. Some have widespread urticaria or few also have chickenpox-like fluidfilled vesicles or blisters. They can have measles-like rashes, Mucormycosis (Black Fungus ) can develop within any part of your body. It may initially occur at the site of skin trauma, but it can quickly spread to another area. $\mathrm{Be}$ on the lookout for symptoms such as: blackened skin tissue, blisters, fever, redness, swelling, tenderness, ulcers.

Complications of mucormycosis include: Blindness Blood clots or blocked vessels Nerve damage

Mucormycosis can be deadly without treatment. Because the infection is so rare, the exact mortality rate isn't clear. But researchers estimate that overall, $54 \%$ of people with mucormycosis die. Figure ( 6 ) \& Figure ( 7 )

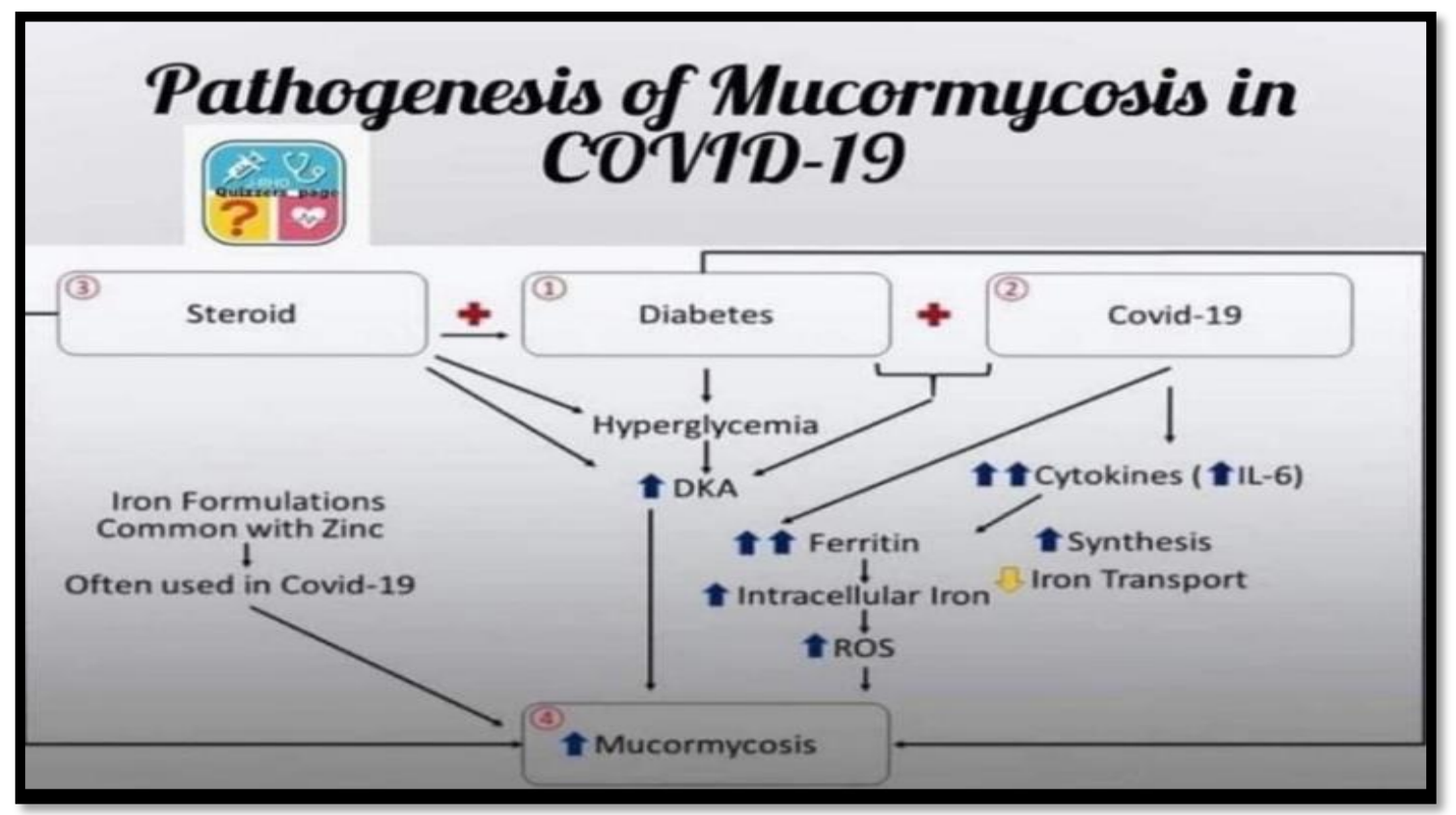

Figure 6 ( Pathogenesis Of Mucormycosis in COVID-19)

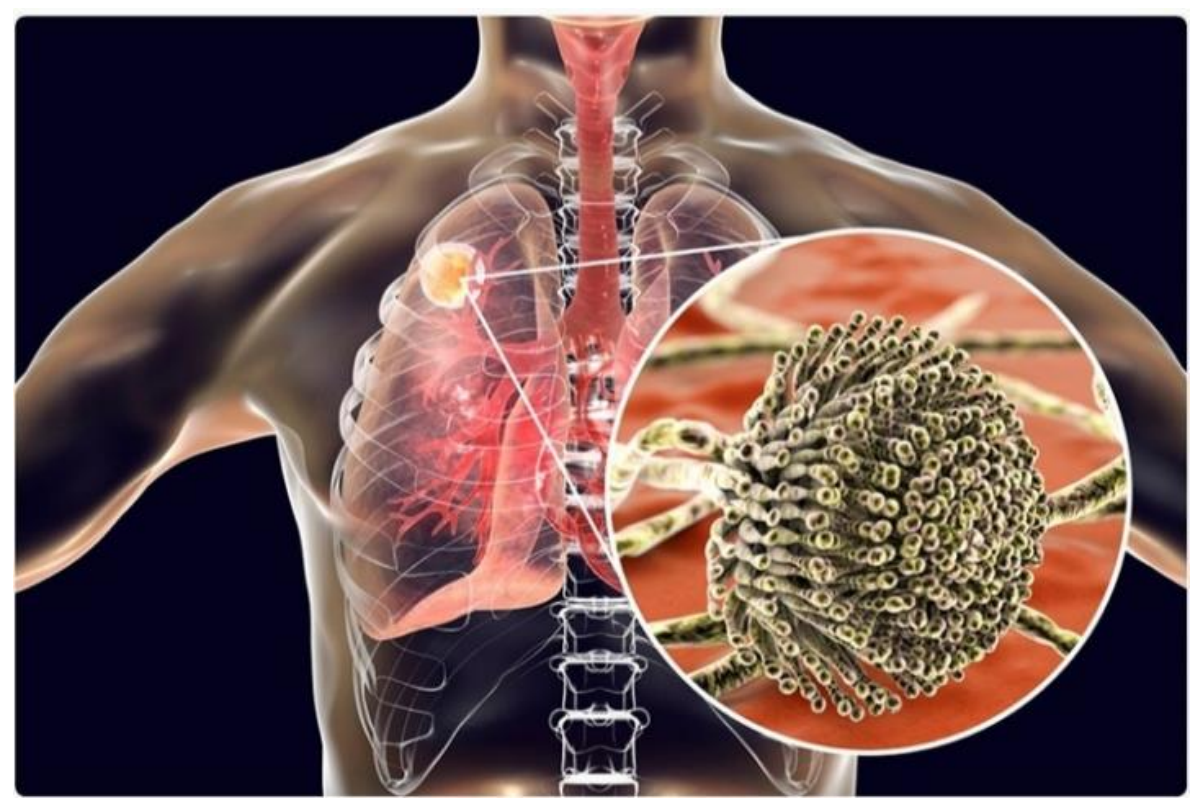

Figure 7 ( Black fungus causes invasive lung infection ) 


\subsection{Precaution and Management}

low-dose systemic corticosteroids as a therapeutic option for COVID-19-associated urticarial rash. Indeed, low-dose systemic corticosteroids, combined with nonsedating antihistamines, can help in managing the hyperactivity of the system in COVID-19, not only to regulate urticaria, but also to boost possibly the survival rate in COVID-19.[22]

Preventions of Black Fungus: keep one's eyes off from areas with lots of dust or soil, like construction or excavation sites. If you have got to be in these areas, wear a mask like an N95. Avoid infected water. this could include floodwater or water-damaged buildings, especially after natural disasters like hurricanes or floods.

If you have got a weakened system, avoid activities that involve dust and soil, like gardening or yard work. If you cannot, protect your skin with shoes, gloves, long pants, and long sleeves. Wash cuts or scrapes with soap and water as soon as you'll. Figure ( 8 )

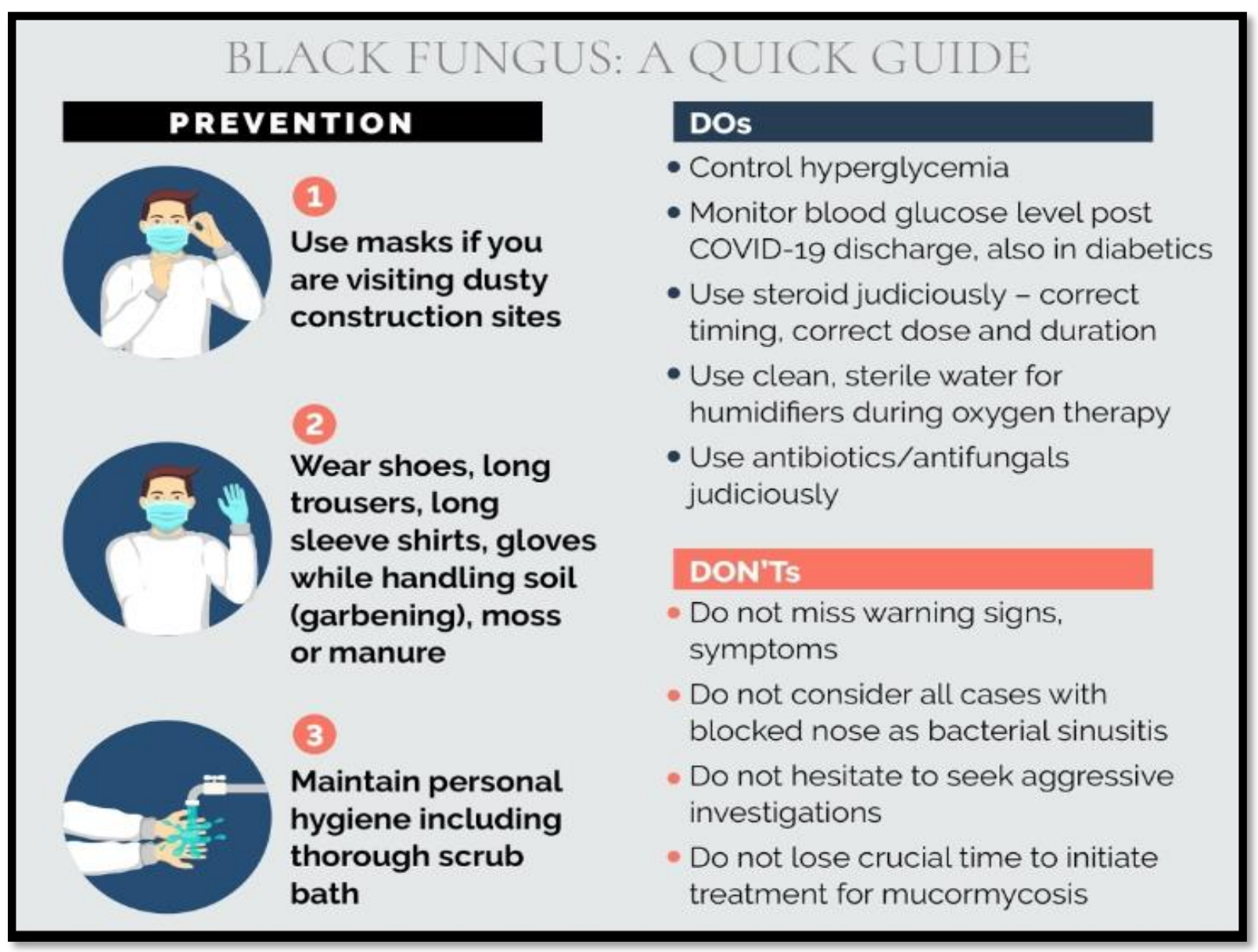

Fig 8 Black Fungus A Quick Guide

\subsection{Psychological effects:}

Because of financial pressure and social isolation many psychological problems can arise. They can be delayed by months. There is an increase in "deaths of despair" from substance misuse or suicide. The risk is greater among persons with dementia. After discharge from ICU, a $30 \%$ of the patients inattention, disorientation problems, or poorly movements in response to questions [23]. Some patients who recover suffering from mental health problems [24]. Like anxiety, major depression episodes, post-traumatic stress disorder (PTSD). Long effects can include development of Alzheimer's or Parkinson's disease.

\subsection{Precaution and Management:}

Healthy Ways to address Stress: Take breaks from watching, reading, or taking note of news 
stories, including those on social media. It's good to learn, but hearing about the pandemic constantly is upsetting. Consider limiting news to simply a pair times each day and disconnecting from phone, TV, and computer screens for ages. Try to eat healthy, well-balanced meals. Exercise regularly. Get lots of sleep. Avoid excessive alcohol, tobacco, and substance use. Make time to unwind. attempt to do another activities you enjoy. Connect with others. Talk with people you trust about your concerns and the way you're feeling. Connect along with your community- or faith-based organizations. While social distancing measures are in situ, try connecting online, through social media, or by phone or mail. Helping Others Cope.

\subsection{Sexual Effects:}

COVID-19 is also related to male ( ED ), factors which will cause the potential onset of (ED) in men.

Vascular effects. ( ED ) may be a predictor of heart condition, so we all know that the system and system are connected. We also know that COVID-19 can cause hyper inflammation throughout the body, especially within the heart and surrounding muscles. Blood supply to the penis can become blocked or narrowed as a results of a replacement or worsened vascular condition caused by the virus.

Psychological impact. gender is closely related to psychological state. The stress, anxiety and depression caused by the virus and pandemic are often linked to sexual dysfunction and poor mood.

Overall health deterioration. erectile dysfunction is often a signal of an underlying problem. Men with poor health are at greater risk for developing erecticle dysfunction and also for having a severe reaction to COVID-19. Since the virus can cause general poor health

is cause for concern both for erecticle dysfunction and other complications in the sexual relationship. Figure ( 9 )

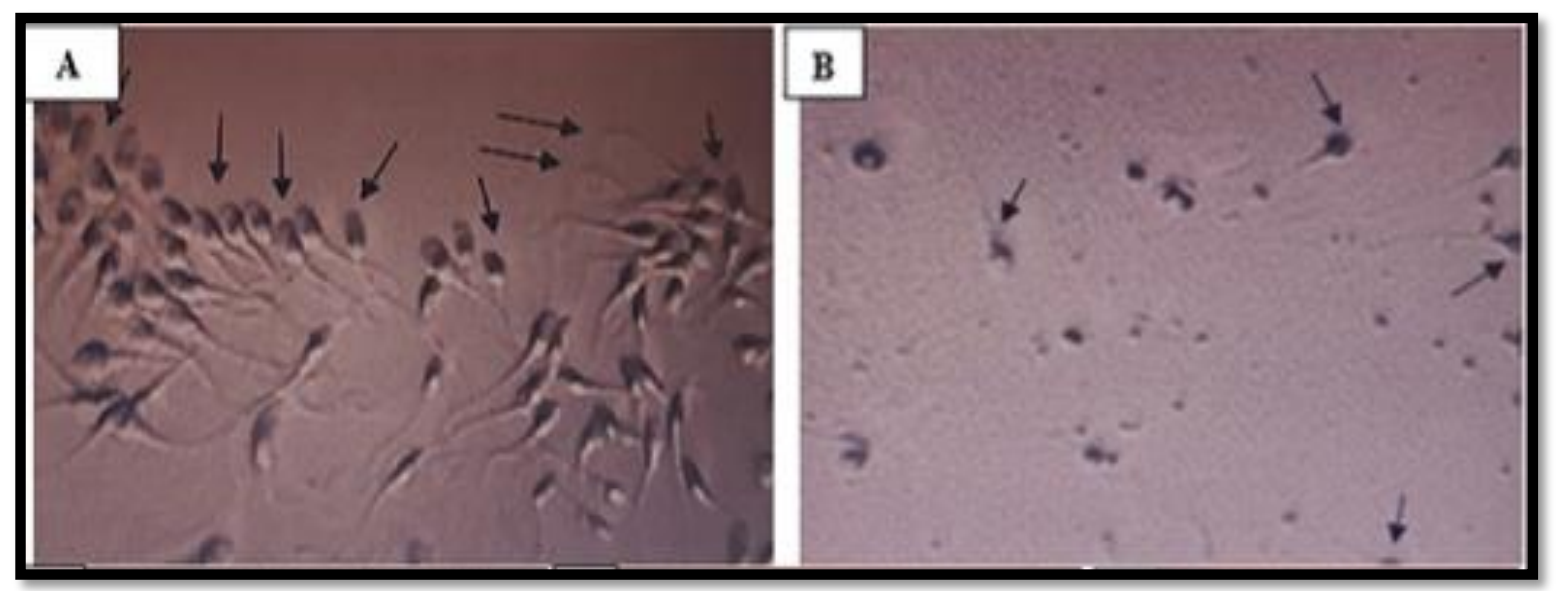

Figure ( 9 ) (A) Normozoospermia with an excellent sperm count, motility and normal morphology in man before acquiring COVID-19. (B) Oligo astheno teratozoopsermia with decreased sperm count, motility and normal morphology in man after acquiring COVID-19.

\subsection{Precaution and Management:}

Those who are stricken by male erecticle dysfunction as a results of Long Covid should be able to access the mandatory treatment. Occasionally, short courses of medicines like low dose Tadalafil or occasional Viagra may help more rapid recovery. and would try and adopt as healthy a way of life limit your alcohol content, stop smoking, eat a varied diet and drink many water to confirm you're well hydrated. try and do a small amount of sunshine exercise day after day to figure on your stamina and breathing. Even a 15-minute walk outside will suffice. you'll want to also cross-check other ways of getting 
sex that doesn't involve any tiring positions within the relationship.

\subsection{In vitro fertilization (IVF) Effects:}

The possibility of the COVID-19 affecting the sperm functions and thr egg performances cannot be excluded. In addition to this an indirect effect of the COVID-19 on gametes and embryos during their manipulation cannot be ruled out.

\subsection{Precaution and Management:}

The European Society for Human Reproduction and Embryology and American Society for Reproductive Medicine, have provided recommendations and guidance to beat and flatten the growing curve of infection in patients who undergo IVF treatments. fertility treatments are postponed so as to support healthcare systems by avoiding placing them under additional stress.Society for Human Reproduction and Embryology and American Society for Reproductive Medicine, have provided recommendations and guidance to beat and flatten the growing curve of infection in patients who undergo IVF treatments. fertility treatments are postponed so as to support healthcare systems by avoiding placing them under additional stress. ${ }^{[25]}$

Finely, COVID-19 vaccination is now offering a way to transition out of this phase of the pandemic.COVID-19 vaccination keep you from getting infected by COVID-19 also keep you from getting seriously ill even if you got COVID19. Getting vaccinated yourself also protect people around you, Once you are fully vaccinated, you can start doing more you should keep using all the tools available to protect yourself and others until you are fully vaccinated. Also it's an easy and safe way protection and the risk of severe illness or death from COVID-19 far outweighs any benefits of natural immunity. The vaccinations will protect you by creating an antibody response without having to experience sickness. And will be an important tool to stopping the pandemic as none of the COVID-19 vaccines can make you infected with COVID19. ${ }^{[26]}$

\section{Conclusion}

Long COVID-19 is a serious problem as the virus binds to ACE2 receptors present all over the body and can affect virtually every system of the body. It can cause cytokine storm which can lead to deaths. Different organs may be affected. viral load and Inflammation, platelet activation, hypercoagulability, endothelial dysfunction constriction of blood vessels, stasis, hypoxia, and muscle immobilization contribute to the complications. The respiratory system is commonly affected. coronary syndrome and congestive heart failure, myocarditis may be present. Patients who are on angiotensin converting enzyme inhibitors and angiotensin II receptor blockers should continue. AKI is usually secondary to systemic disturbances. Meningitis, encephalitis, encephalopathy, stroke, and delirium are also observed. Impaired sense of smell and taste are observed. Eyes can also be sources of viral infection. Mentally problems are common among patients as well as providers. Gl symptoms are observed. Patchy skin erythematous rash is commonest skin manifestation. Thus, COVID-19 can affect virtually any organ in the body, so vaccinations are the smart way to control our lives and ending this disaster.

Data availability statement: The original contributions generated for the study are included within the article/supplementary materials, further inquiries are often directed to the corresponding author.

Conflict of interest: The author declare that the research was conducted within the absence of any commercial or financial relationships that might be construed as a possible conflict of interest.

Author contributions: the author approved the ultimate manuscript .

\section{Acknowledgments:}

I wish to present my special thanks to my lovely hospital El-Abbassia Mental Hospital - EGYPT, with a virtual platform of young researchers for their support during the preparation of the paper. 


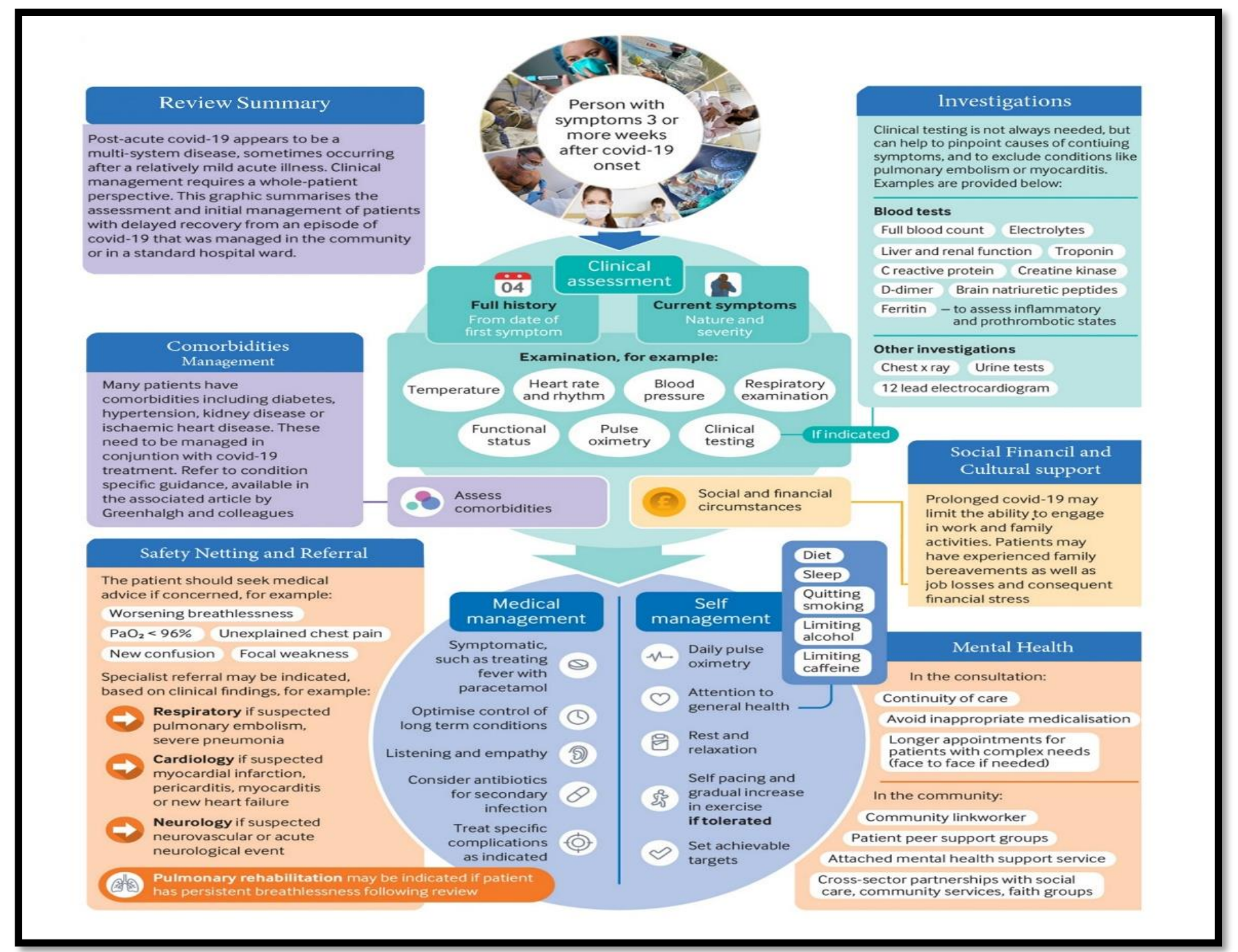

Fig 10 Summary of long COVID-19 precaution \& management

\section{References:}

[1]. How does coronavirus kill? Clinicians trace a ferocious rampage through the body, from brain to toes. [Jun;2020 ];Wadman M, Couzin-Frankel J, Kaiser $J$, at al. https://www.sciencemag.org/news/2020/04/howdoes-coronavirus-kill-clinicians-trace-ferociousrampage-through-body-brain-toes 2020 .

[2]. National Institute for Health and Care Excellence (NICE). COVID-19 Rapid Guideline: Managing The Long-term Effects of COVID-19. 2020 December 18 [Accessed 8 January 2021].

[3]. Endothelial cell infection and endotheliitis in COVID-19. Varga Z, Flammer A, Steiger P, et al. Lancet. 2020;395:1417-1418. [PMC free article] [PubMed] [Google Scholar].

[4]. Coronavirus disease 2019 (COVID-19) treatment guidelines.

[Jun;2020 ];https://covid19treatmentguidelines.nih.gov 2020

[5]. Surviving sepsis campaign: guidelines on the management of critically ill adults with coronavirus disease 2019 (COVID-19) Alhazzani W, Møller M,
Arabi Y, et al. Intensive Care Med. 2020;46:854887. [PMC free article] [PubMed] [Google Scholar] [6]. COVID-19 autopsies, Oklahoma, USA. Barton L, Duval E, Stroberg E, et al. Am J Clin Pathol. 2020;153:725-733. [PMC free article] [PubMed] [Google Scholar]

[7]. Pathological findings of COVID-19 associated with acute respiratory distress syndrome. Xu Z, Shi L, Wang Y, et al. Lancet. 2020;8:420-422. [PMC free article] [PubMed] [Google Scholar]

[8]. Time to consider histologic pattern of lung injury to treat critically ill patients with COVID-19 infection. Copin M, Parmentier E, Duburcq T. Intensive Care Med. 2020;46:1124-1126. [PMC free article] [PubMed] [Google Scholar]

[9]. Management of COVID-19 respiratory distress. Marini J, Gattinoni L. JAMA. 2020 [PubMed] [Google Scholar]

[10]. Association of noninvasive oxygenation strategies with all-cause mortality in adults with acute hypoxemic respiratory failure. A systematic review and meta-analysis. Ferreyro B, Angriman 
F, Munshi L, et al. JAMA. 2020;324:1-12 [PMC free article] [PubMed] [Google Scholar]

[11]. https://www.brit-

thoracic.org.uk/document-library/qualityimprovement/covid-19/resp-follow-up-guidancepost-covid-pneumonia/

[12]. Potential effects of coronaviruses on the cardiovascular system: a review. Madjid M, Safavi-Naeini P, Solomon S, et al. JAMA Cardiol. 2020 [PubMed] [Google Scholar]

[13]. COVID-19 and the heart. Akhmerov A, Marbán E. Circ Res. 2020;126:1443-1455. [PMC free article] [PubMed] [Google Scholar]

[14]. Management of patients on dialysis and with kidney transplantation during the SARS-CoV-2 (COVID-19) pandemic in Brescia, Italy. Alberici F, Delbarba E, Manenti C, et al. Kidney Int Rep. 2020;5:580-585. [PMC free article] [PubMed] [Google Scholar]

[15]. Neurologic features in severe SARS-CoV-2 infection. Helms J, Kremer S, Merdji $\mathrm{H}$, et al. N Engl J Med. 2020;382:2268-2270. [PMC free article] [PubMed] [Google Scholar]

[16]. COVID-19 pandemic: a neurological perspective. Lahiri D, Ardila A. Cureus. 2020;12:0. [PMC free article] [PubMed] [Google Scholar]

[17]. Neurologic manifestations of hospitalized patients with coronavirus disease 2019 in Wuhan, China. Mao L, Jin H, Wang M, et al. JAMA Neurol. 2020;77 :1-9. [PMC free article] [PubMed] [Google Scholar]

[18]. Large-vessel stroke as a presenting feature of Covid-19 in the young. Oxley T, Mocco J, Majidi S, et al. N Engl J Med. 2020;382:0. [PMC free article] [PubMed] [Google Scholar]

[19]. Alterations in smell or taste in mildly symptomatic outpatients with SARS-CoV-2 infection. Spinato G, Fabbris C, Polesel J, et al. JAMA. 2020;323:2089-2090. [PMC free article] [PubMed] [Google Scholar]

[20]. Characteristics of ocular findings of patients with coronavirus disease 2019 (COVID19) in Hubei province, China. Wu P, Duan F, Luo C, et al. JAMA Ophthalmol. 2020;138:575-578. [PMC free article] [PubMed] [Google Scholar]

[21]. Cutaneous manifestations in COVID- 19: a first perspective. Recalcati S. J Eur Acad Dermatol. 2020; 34:0. [PubMed] [Google Scholar]

[22]. Covid-19 pandemic and the skin - what should dermatologists know? Darlenski R, Tsankov N. Clin Dermatol. 2020 [Google Scholar] [23]. Neurologic features in severe SARS-CoV-2 infection. Helms J, Kremer S, Merdji $\mathrm{H}$, et al. $\mathrm{N}$ Engl J Med. 2020; 382:2268-2270. [PMC free article] [PubMed] [Google Scholar]
[24]. Mental health and the Covid-19 pandemic. Pfefferbaum B, North C. N Engl J Med. 2020 [Google Scholar] ${ }^{*}$ Data Availability Statement:

[25]. Rodriguez-Wallberg KA, Wikander I. 2020. A global recommendation for restrictive provision of fertility treatments during the COVID19 pandemic. Acta Obstet. Gynecol. Scand. Apr 8

[26]. https://www.cdc.gov/coronavirus/2019-ncov/ vaccines/vaccine-benefits.html

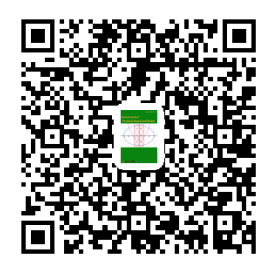

